\title{
La teoría keynesiana y su alcance frente a la crisis de las naciones: caso Ecuador
}

\section{Keynesian theory and its scope in the face of the crisis of nations: case Ecuador}

\author{
Holger Fabrizzio Bejarano Copo \\ Universidad Técnica de Machala, Ecuador \\ Sylvia Jacqueline Mosquera Maldonado \\ Universidad Técnica de Machala, Ecuador \\ Gregory Darío Varela Veliz \\ Universidad Técnica de Machala, Ecuador \\ Diego Armando Martínez Jiménez \\ Universidad Técnica de Machala, Ecuador
}

Autor para correspondencia: hbejarano@utmachala.edu.ec, smosquera@utmachala.edu.ec, gredavave@hotmail.com,diamartinez2010@hotmail.com Fecha de recepción: 17 de octubre de 2017 - Fecha de aceptación: 15 de Abril de 2018

Resumen: En este artículo se revisa la base teoría keynesiana para poder explicar las decisiones macroeconómicas tomadas por el gobierno nacional a partir del año del 2007, en cuanto a la fuerte figura institucional en la actividad económica del Ecuador. Como paradigma dominante en siglos pasados, el keynesianismo ha sido el modelo económico adoptado para combatir las diferentes crisis de las naciones, las que se afirman cada cierto tiempo que son resultado de la corriente liberal que reduce a su mínima expresión el tamaño del estado. Frente a esto, los gobiernos justifican su intervención para poder tratar de reducir la inequidad redistributiva que afirman ser producida por, la ortodoxia liberal, que se acentúa cada cierto tiempo.

Palabras Claves: teoría de Keynes; crisis económica; teoría neoclásica; crecimiento económico; Ecuador

\begin{abstract}
In this article, we review the Keynesian theory base in order to explain the macroeconomic decisions taken by the national government as of 2007, regarding the strong institutional figure in economic activity in Ecuador. As a dominant paradigm in past centuries, Keynesianism has been the economic model adopted to combat the different crises of nations, which are asserted from, time to time that are the result of the liberal current that reduces to a minimum the size of the state. Against this, governments justify their intervention in order to try to reduce the redistributive inequities that claim to be produced by liberal orthodoxy that is accentuated from time to time.
\end{abstract}

Key words: theory of Keynes; economic crisis; neoclassical theory; economic growth; Ecuador 


\section{Introducción}

Dentro del ámbito económico nacional, las ideologías implantadas por Keynes, dieron lugar al legado de la generalización de la presencia del Estado en los mercados, y la labor en la redistribución y la estabilización, “en base al Estado del Bienestar, la cual fue más desarrollado en la Europa Occidental que en los Estados Unidos o Japón, tras la Segunda Guerra Mundial” (Galarza, 1997).

Como lo menciona, Galarza (1997) la ideología económica keynesiana es un ataque de doble vía, a la doctrina económica que se establece desde la perspectiva microeconómica, a la diferencia del esquema de oferta y demanda global, además de oponerse a la ideología del mercado libre, lo que llama el espacio para la intervención del Estado como una guía en la dirección de las fuerzas económicas para lograr el pleno empleo y la estabilidad de los precios.

La ideología que está destinado a ser utilizado desde el diseño del estado de bienestar para hacer frente a la crisis económica o la recesión, que atraviesa el actual gobierno de Ecuador, que se dice por los expertos que está en una etapa inicial, sin embargo, otros analistas dicen que es un problema que se ha producido desde la caída de los precios del petróleo desde 2015.

Esta crisis, no declarada como tal, requiere una revisión de las medidas de política económica en el gobierno nacional, porque las consecuencias para el empleo, el producto interno bruto, la deuda, importaciones, etc, se han convertido en un problema en el análisis de esta, más allá de más de la misma acción del gobierno, cuya acción es muy decisiva en la política pública con una clara intervención en las actividades productivas.

Y aunque la teoría neoclásica ha tratado de negar el carácter revolucionario que adquiere Keynes en su posición teórica, es igualmente cierto el reconocimiento de su trabajo como otros teóricos se dieron cuenta; En particular, y en la estela de la "revolución keynesiana", la opinión de Archibal es que el keynesiano no reemplace a la teoría clásica, debido a una refutación de Popper, pero "debido a la creciente preocupación y la falta de satisfacción producida por los fenómenos que no se explicó " (Montoya, 2009).

En este sentido, la política económica de la prosperidad excesiva del gobierno nacional desde el año 2007 hasta la fecha, con el apoyo de la impresionante cifra de precios del barril de petróleo por encima de 100 dólares, tiene un descenso que muestra con claros signos de desaceleración económica, tomemos por ejemplo la tasa de desempleo registrada al 31 de marzo 2016 un 7,35 \% por parte del Banco Central del Ecuador (BCE), el más alto desde 2011, otros indicadores proporcionados por la misma institución están reportando un declive del PIB en $1,9 \%$ en el primer trimestre del mismo año, hay indicadores que confirman la entrada en una crisis económica. (Banco Central del Ecador, 2016)

\section{Fundamentación de la aplicación teórica Keynesiana}

La Teoría Keynesiana ha sido objeto de varios estudios, en la tabla 1, mencionaremos diferentes autores que presentan su enfoque en base a ella, estos adaptan su investigación de forma cronológica. 
Tabla 1. Revisión Literaria de la Teoría Keynesiana

\begin{tabular}{|c|c|c|}
\hline Autor & Tema & Definición \\
\hline $\begin{array}{l}\text { Axel } \\
\text { Leijonhufvud } \\
(1987)\end{array}$ & $\begin{array}{l}\text { ¿Qué le ha sucedido } \\
\text { a la economía } \\
\text { keynesiana? }\end{array}$ & $\begin{array}{l}\text { Los métodos empíricos keynesianos que se utilizaron la fecha, no dieron } \\
\text { resultados sobre el cambio en el ámbito monetario, ni señalaron la } \\
\text { dirección viable a tomarse. Un énfasis menor a la econometría y mayor } \\
\text { al conocimiento de la historia, hubiera ayudado de mejor manera. }\end{array}$ \\
\hline $\begin{array}{l}\text { Esteban Pérez } \\
\text { Caldentey } \\
(\mathbf{2 0 0 3 )}\end{array}$ & $\begin{array}{l}\text { Chicago Keynes } \\
\text { and Fiscal Policy. }\end{array}$ & $\begin{array}{l}\text { El enfoque de la Teoría Keynesiana, era una parte integral del marco } \\
\text { teórico de la Teoría General del Empleo, Intereses y Dinero, ya que, la } \\
\text { política fiscal se preocupaba de mantener la estabilidad de la inversión } \\
\text { en la escasez de capital. Esto requirió la planificación y la elaboración } \\
\text { de un programa a largo plazo. Además de, la identificación de los } \\
\text { déficits pendientes como herramienta de estabilización de política fiscal } \\
\text { de corto plazo. }\end{array}$ \\
\hline $\begin{array}{l}\text { Henri Huoben } \\
\text { (2011) }\end{array}$ & $\begin{array}{l}\text { A Marxist critic on } \\
\text { the Keynesian } \\
\text { analysis of the } \\
\text { economic crisis. }\end{array}$ & $\begin{array}{l}\text { Las políticas de la teoría keynesiana pueden ser una solución a corto } \\
\text { plazo de problemas, pero si persisten pueden ser peores que el problema } \\
\text { en sí, en vista que pone en marcha una sociedad basada en la igualdad y } \\
\text { la solidaridad que garantiza los intereses de la mayoría a través de una } \\
\text { planificación centralizada. En contraste, las políticas de competitividad } \\
\text { de los clásicos, permiten a la economía recuperarse, pero a expensas de } \\
\text { los demás, lo cual, puede presentar un ambiente de tensión y de } \\
\text { conflictos. }\end{array}$ \\
\hline $\begin{array}{l}\text { Federico Novelo } \\
\text { (2016) }\end{array}$ & $\begin{array}{l}\text { La Pertinencia } \\
\text { actual de la Teoría } \\
\text { General de Keynes. }\end{array}$ & $\begin{array}{l}\text { El keynesianismo es una semilla que no puede ser trasplantada en suelo } \\
\text { extraño, si así se hace, esta muere y antes de morir envenena su entorno. } \\
\text { La teoría general de Keynes, promueve el conservacionismo económico, } \\
\text { también la expansión monetaria, la política fiscal, especialmente el } \\
\text { Estado del Bienestar, apoyada por la interpretación monetarista y los } \\
\text { individuos en el ciclo económico. }\end{array}$ \\
\hline
\end{tabular}

Elaborado por: Los Autores

En la tabla anterior se visualizan varios autores, que en las últimas décadas han facilitado sus diferentes puntos de vista con respecto a la teoría keynesiana y sus implicaciones en la economía a través del tiempo.

\section{La crisis económica y el Ecuador}

Debemos tener en cuenta que la crisis ecuatoriana tiene su presencia en el gobierno constitucional del Presidente Econ. Rafael Correa Delgado, y para muchos autores, su inicio internacional se dio en agosto de 2007 en el sector inmobiliario de Estados Unidos, debido al fracaso de las hipotecas de alto riesgo, una situación que se ha prestado poca atención en toda la economía, no sólo de este países, sino también a nivel mundial.(López Arévalo \& Peláez Herreros, 2015)

La tendencia cada vez mayor en la financiación de las economías y la hegemonía que el capital especulativo se llevó a sus preocupaciones con el concepto de liquidez analizada por Keynes (Venegas \& Pilar, 2010). Para ellos, "la liquidez de los mercados de inversión facilita a menudo, pero a veces impide, el curso de las nuevas inversiones, ya que el hecho de que cada inversor individual es la ilusión de que su compromiso es 'líquido' (a pesar de que no es cierto para todos los inversores colectivamente) calmar sus nervios y estimular más a asumir el riesgo. "Por otra parte," si las compras individuales de inversión perdieron su liquidez, esto podría bloquear seriamente nuevas inversiones como las formas alternativas individuales para mantener sus ahorros (Venegas \& Pilar, 2010). 
En este contexto, el Ecuador es un país estrechamente vinculado al sistema productivo, financiero y comercial estadounidense a través de los sistemas de crédito, las preferencias arancelarias, que también han sentido el impacto de la crisis por la desaceleración y recesión posterior del líder mundial. El uso de un fondo de estabilización ha permitido que el país no se sienta muchos los efectos de la crisis, se pueden demostrar en el análisis de las cifras macroeconómicas del Banco Central, que en los años más complicados el Producto Interno Bruto (PIB) aumentó de 24,032 a 24,119.5 millones de dólares, un ligero aumento, pero crecimiento al fin de la crisis global en años (hay que señalar que el impacto de esta crisis no fue homogéneo entre naciones). Las consecuencias de la tribulación económica se produjeron de una manera y con diferentes intensidades de los otros menos relacionada con las áreas de operaciones de Estados Unidos.

La presencia de los ciclos económicos y la crisis asociada es una realidad que no permite el cuestionamiento, ya que, para los economistas, era obvio que en ocasiones hemos visto que tienen ciclos de nombres como: Kondratieff, Clement Juglar, Kuznets, etc. El análisis de la crisis lleva a una mayor presencia durante la Gran Depresión y las sucedidas hasta ahora. La última sobre el sistema de crédito de Estados Unidos, durante el verano de 2007, con un impacto en la economía. En ese momento, las primeras declaraciones del gobierno entrantes tendían a minimizar el impacto potencial en el país.

Giraldo (2007), determina un recuento interesante, cuenta de la existencia del fondo de estabilización como antecedentes a Ecuador respuesta de una buena gestión antes la crisis global, mencionó que la renegociación de los contratos petroleros era un buen punto de lograr una distribución equitativa de beneficios extraordinarios del petróleo entre las empresas y el estado. El gobierno de la revolución ciudadana, el 3 de abril de 2008 emitió la ley orgánica para la recuperación de la utilización de los recursos petroleros del estado y la racionalización de los procesos administrativos de endeudamiento.

La ley descartó todos los fondos que eran sostenidos con los recursos petroleros en sus artículos 2, 3 y 13, y por el otro, diseñaba la eliminación de los requerimientos para el convenio de deuda. (Asamblea Constituyente, 2008) Los antecedentes de la intervención del gobierno de carácter muy activa en la gestión pública, una transferencia de las acciones privadas a lo público. Buiter (1977) determina que el efecto desplazamiento, se describe al traslado de la actividad económica privada a la actividad económica pública. Esto se lo conoce como el "desvío" de Keynes (1929), y señala que este tema tiene una larda data en la teoría política y el debate macroeconómico.

La inflexibilidad de los presupuestos públicos de la región, junto con las políticas neoliberales de rigideces, obligaron a los gobiernos a hacer frente a los pagos de la deuda, constituían una restricción en la gestión de la política económica en general y de la fiscal, en donde los gobiernos poseen pocas posibilidades para establecer, políticas económicas.

La tabla 2 muestra la evolución del presupuesto general del Estado desde 2007, despega en 2009 con el año de la crisis en respuesta a la intervención gubernamental descrita por la CEPAL, con un aumento del 121, 32 por ciento, con altos niveles de inversión pública. 


\begin{tabular}{|c|c|c|}
\hline Año & Presupuesto Inicial & \% Incremento Presup. Inicial \\
\hline 2007 & $9,768.00$ & \\
\hline 2008 & $10,358.00$ & $6.04 \%$ \\
\hline 2009 & $22,924.00$ & $121.32 \%$ \\
\hline 2010 & $21,282.00$ & $-7.16 \%$ \\
\hline 2011 & $23,950.00$ & $12.54 \%$ \\
\hline 2012 & $26,109.00$ & $9.01 \%$ \\
\hline 2013 & $32,366.00$ & $23.96 \%$ \\
\hline 2014 & $34,300.00$ & $5.98 \%$ \\
\hline 2015 & $36,317.00$ & $5.88 \%$ \\
\hline
\end{tabular}

\section{Fuente: Ministerio de Finanzas}

Esta acción en una forma de crítica del neoliberalismo que indica fracaso incluyendo la estrategia de crecimiento, bienestar y prosperidad; y como un proyecto de clase ha demostrado un enorme éxito para la movilización de capital, poder y riqueza. Para los gobiernos con una tendencia keynesiana a reducir las desigualdades sociales, es un costo que no es soportado por la ortodoxia, que no puede ser ignorada o fácilmente mitigada por la implementación de políticas de "reducción de la pobreza", especialmente en los países. Márquez Covarrubias (2016) sugiere que la planificación a largo plazo debería ser más necesaria en el diseño de políticas públicas de alto impacto para reducir las desigualdades sociales.

Algunos autores sostienen que el gasto expansionista del gobierno, no es un mal en sí mismo; en muchos casos refleja la expansión progresiva de los derechos económicos y sociales, es un medio para restablecer los beneficios sociales públicos con participación directa, y en otros el crecimiento es el producto de la implementación gradual de las reformas económicas y la importancia social, en el Ecuador el caso de las inversiones en sectores sociales como la educación, la salud, las carreteras, etc.

El grado de inflexibilidad en el presupuesto de un país puede ser justificado por razones teóricas y prácticas, excesiva rigidez de los costes y los recursos pueden tener una serie de efectos negativos, muchos de ellos asociados con las órdenes organizaciones internacionales como el Fondo monetario Internacional y el Banco Mundial.

En este sentido, se afirma que los gobiernos deben superar múltiples obstáculos para la asignación del presupuesto en el caso de Ecuador, el gobierno consolida el apoyo a una propuesta de defensa a la social, con mayoría legislativa que da paso a una la nueva constitución le da legitimidad a las políticas de reforma requeridos y permite que sean financiados sectores de la sociedad con mayores recursos, a través de reformas fiscales que amplíen la base de contribuyentes e identificar nuevos grupos económicos, como verse en la Figura 1, también se determinó el coeficiente de determinación del 96\%. 


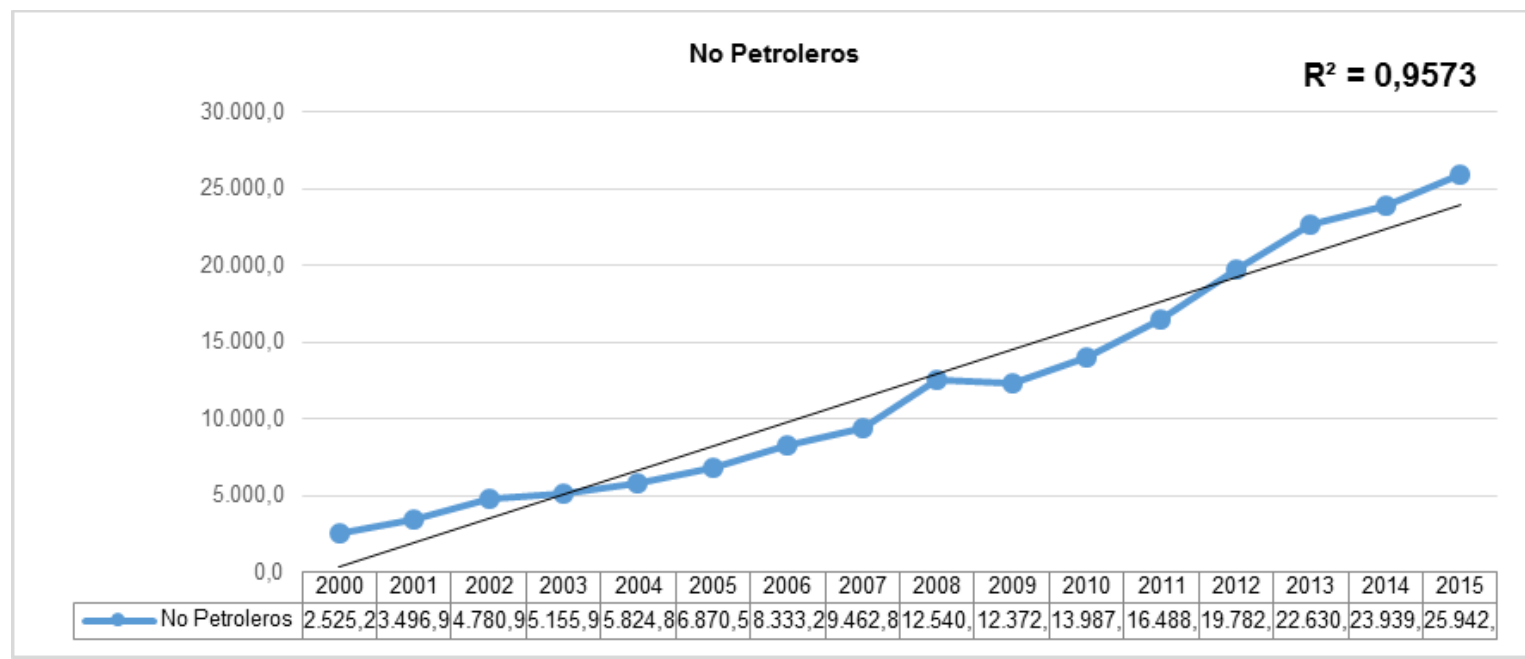

Grafico 1. Ingresos No Petroleros

Fuente: Banco central del Ecuador

Elaboración: Los autores

Dentro de estos últimos diez y seis años, se ha demostrado que el crecimiento de los ingresos no petroleros como lo son: el IVA, el ICE, el impuesto a la renta, los aranceles, etc., han tenido un crecimiento paulatino, desde el 2007 al 2015 en donde la recaudación fiscal casi se duplica, con lo cual el gobierno cuenta con el dinero para tratar de impulsar las políticas públicas en torno al desarrollo socioeconómico que ayuden a la reconstrucción de una nueva política fiscal que promueva cohesión social y desarrollo económico en Ecuador.

Es por eso que se debe mejorar la distribución del presupuesto para asegurar el acceso de la ciudadanía a los beneficios o prestaciones sociales, en forma eficaz y eficiente al mínimo costo, en base a los estándares de calidad, solidaria y oportuna, para ello se debería programan leyes que puedan promover la descentralización y desconcentración del gobierno central.

El ímpetu de tratar de satisfacer las necesidades básicas de la sociedad, demanda de una de tener una buena distribución y equitativa de la riqueza, que sólo se puede alcanzar cuando exista una amplia participación de la sociedad en su producción. En tal sentido, es esencial que el entendimiento que el estado no puede saberlo todo, pero tampoco el mercado es un recurso infalible. (Cuesta Caza, 2013)

No estamos hablando sólo de crecimiento económico o del PIB; hablamos de amplias relaciones entre los seres humanos, la naturaleza, la vida comunitaria, los antepasados, el pasado y el futuro. Las alternativas son la construcción y definición de pueblos en los procesos de cambio. Estos, a su vez transformadores, en sujetos de un proceso cada vez más social mediante la introducción de un nuevo paradigma de desarrollo: el Buen Vivir.(Cuesta Caza, 2013; Secretaría Nacional de Planificación y Desarrollo, 2013, p.32-33)

En este sentido, las herramientas de política fiscal, especialmente los impuestos, deberían ser una clave para reducir las desigualdades y lograr el elemento de justicia social. En Ecuador, el papel del fuerte estado de la subida de la izquierda en 2007, hace hincapié en el despliegue de reformas tributarias en total trece hasta la fecha, que ennoblece la escalada progresiva y equidad, manifestada por funcionarios del gobierno. Como se verá más adelante, esto se presenta entre 2007-2012 indicadores de mejora de la desigualdad per cápita; el estrechamiento de la brecha 
entre ricos y pobres y una mejora en la percepción de personas que se asemejan a la redistribución más equitativa de la riqueza actual en el país.

Horton \& El-Ganainy (2012) explican que la política fiscal es el uso del gasto público y los impuestos para intervenir en la economía. Los gobiernos suelen utilizar la política fiscal para promover el crecimiento sostenible y reducir la pobreza. El papel y los objetivos de la política fiscal han ganado importancia durante la reciente crisis económica mundial, para ayudar a los grupos para reducir la pobreza a través de subsidios o que reduzca los impuestos específicos.

Para Keynes, es esencial la acción del gobierno, no sólo o primordialmente para corregir los resultados distributivos adversos, sino que también y sobre todo para mantener la demanda efectiva total y, por lo tanto, el pleno empleo y la producción completa (Streeten, 2016).

\section{Las finanzas públicas en Ecuador}

Un breve análisis histórico de las finanzas públicas en el Ecuador demuestra una alta vulnerabilidad a factores externos, como las fluctuaciones en el precio del petróleo, los fenómenos naturales, los conflictos bélicos, entre otros; y a factores internos como las inflexibilidades del gasto y las crecientes presiones de gasto fiscal. (Almeida, 2009)

La adaptación del dólar como moneda oficial, la subida del precio de petróleo, el establecimiento de reglas macrofiscales, han ayudado fundamentalmente a la situación fiscal del Sector Público No Financiero (SPNF), en donde a partir del año 2000 ha tenido resultados positivos, obteniendo así en el año 2006 un superávit del 3,6\% en el PIB, lo que conllevo a reducir la relación deuda/PIB. Pero, aún existen problemas estructurales como son las inflexibilidades fiscales que le imposibilitan al Gobierno Central (GC) poder ejecutar efectivamente sus políticas y ponen en riesgo la sostenibilidad fiscal. (Almeida, 2009)

En el gráfico 2, se demuestra como el gasto total del gobierno toma un crecimiento importante desde el 2007, con base la política del Buen Vivir (Sumak Kausay), con una visión para poder cumplir con los objetivos y lineamientos del PNBV como un reto en especial a lo concerniente con la política fiscal y su uso para la redistribución de la riqueza a mediante los impuestos y la generación de la riqueza a través de la inversión pública, con un coeficiente de determinación del $92 \%$.

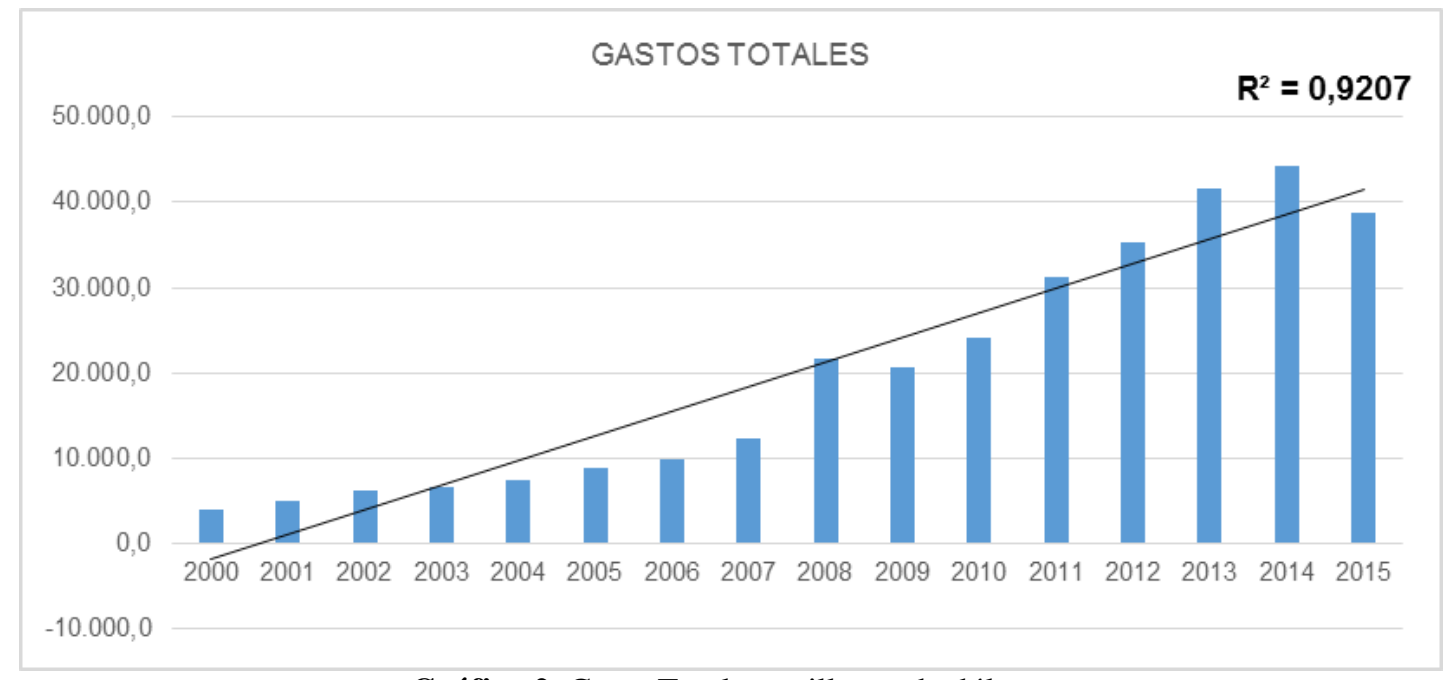

Gráfico 2. Gasto Total en millones de dólares 
Fuente: Banco Central del Ecuador

Elaboración: Los autores

Se puede observar como la intervención del gobierno central, ha toma como base fundamental el PNBV y para ello, toma como directriz a los objetivos: 1 que trata sobre, favorecer la igualdad, cohesión e integración social y el objetivo 11, que busca establecer un sistema económico social, solidario y sostenible (Secretaría Nacional de Planificación y Desarrollo, 2013). Con la cual se está rompiendo paradigmas como las del Fondo Monetario Internacional (FMI) la cual impulsa la subida de la tasa de interés, la reducción del gasto público y el incremento de los impuestos, mecanismos que no dan ninguna clase de resultados positivos y contraindicada por la experiencia histórica para combatir la recesión y el desempleo, que se sigue mucho por quienes piensan más por el mercado capitalista, que por el bienestar social. (Cuello, 1999)

En relación a la crisis histórica económica del Ecuador, se desarrolla un gobierno diferente que se basa en otros lineamientos y que toma a la inversión como la base fundamental para el cambio de la desigualdad social, en el gráfico 3 se puede apreciar como este se ha incrementado en los últimos años, a partir del desde el año 2008 hasta el 2015, siendo explicado por el gobierno del presidente Rafael Correa como la mejor forma de ahorrar a futuro.

En base a la calidad de las acciones fiscales frente a contextos de crisis, la presencia de inflexibilidades afecta negativamente; es por eso que las autoridades tienden como un objetivo primordial, ajustar los gastos de capital,

Pero la eficacia de estas medidas es relativa, ya que son insuficiente para equilibrar el presupuesto y otras partidas de gasto son "rigurosas", como sueldos y salarios en el sector público. Al mismo tiempo, las rigideces presupuestarias pueden limitar la reasignación de los recursos para las redes sociales en tiempos de crisis socioeconómica.(Cetrángolo, Pablo, \& Compiladores, 2009)

Con lo anterior, es posible considerar que "en la etapa de ralentización del ciclo económico en el que se produce una disminución de los ingresos fiscales, el gobierno está obligado y comprometido a asumir estos gastos, que se convierten en la deuda inevitable que perjudica a las finanzas públicas. Por consiguiente, en épocas de austeridad presupuestaria, los gastos "obligatorios" no coinciden necesariamente con las prioridades del gasto público. 


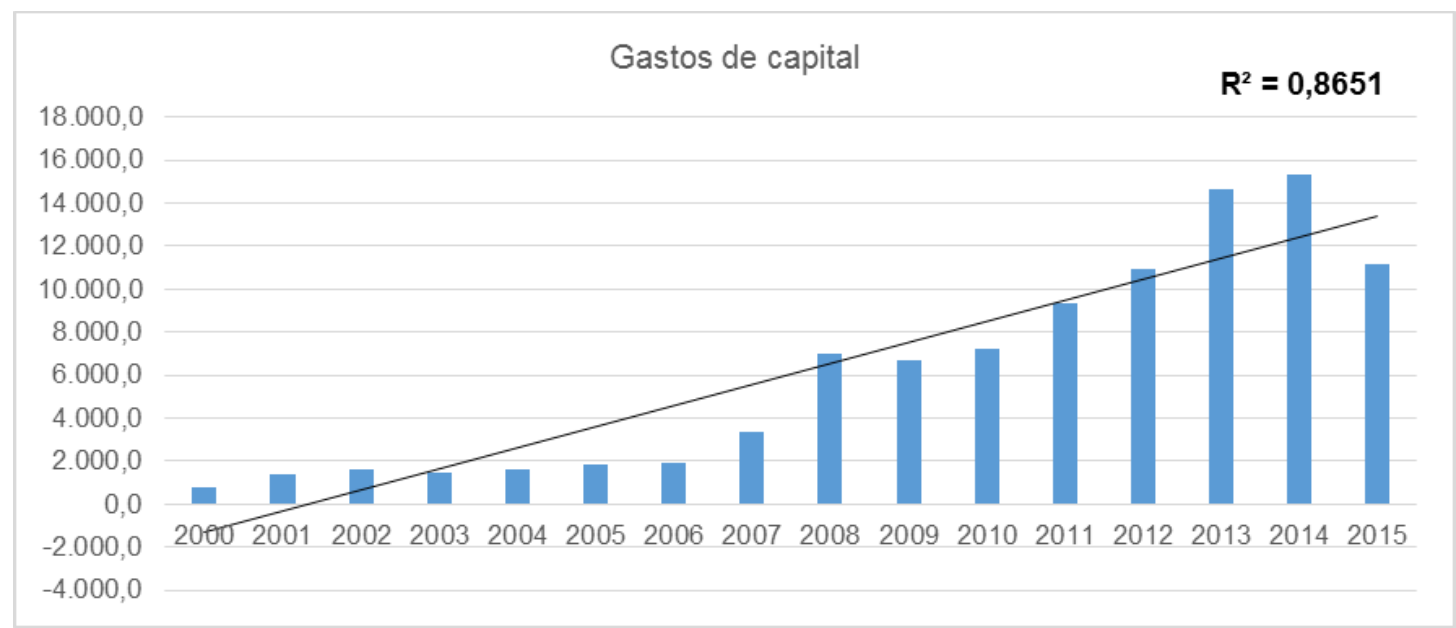

Gráfico 3. Gastos de capital en millones de dólares

Fuente: Banco Central del Ecuador

Elaboración: Los autores

Conclusiones

Se puede determinar que el punto de vista de la para resolver los problemas del “desarrollo, la desigualdad social y la pobreza crónica en América Latina, será esencial el rechazo al capitalismo dependiente, el imperialismo y cortar los lazos con el exterior, tendiendo en el horizonte la construcción del socialismo".(Gutiérrez, 2008)

Ese puede ser el objetivo del actual gobierno y la razón de sus decisiones frente a la crisis, en respuesta al desarrollo de un enfoque humanista que sitúa a la persona, las necesidades, las aspiraciones y capacidades en el centro el esfuerzo de desarrollo. Una propuesta que trasciende los límites del modelo neoliberal en el que se hablaba de los saldos presupuestarios y las finanzas sanas durante toda la década de los ochenta, cuando el déficit se justifica en términos de desarrollo humano basados en el Sumak Kawsay.

Entonces que la mejor forma de acción antes la crisis, es tomar la política fiscal como herramienta de intervención, teniendo en cuenta la política de la pérdida de cambio, tal como se explica en el programa de dolarización adoptada por la economía ecuatoriana en el 2000, los déficits presupuestarios romper la tradición, convirtiéndose en algo habitual que se justifica por la alta inversión pública que intenta abordar la crisis, la creación del pleno empleo y la redistribución de la riqueza.

Por último, podemos decir que "en ausencia de un nuevo Keynes, los economistas y los políticos han recurrido a la vieja teoría de Keynes para dar significado a la crisis económica y sus consecuencias" (De Grauwe, 2010). Incluso como afirma Houben (2011) "John Maynard Keynes sigue siendo una base fundamental para la clave en el pensamiento económico actual. Incluso con el movimiento neoliberal dominando el accionar económico en las últimas décadas, las ideas keynesianas siendo duradera, especialmente en tiempos de recesión".

\section{Bibliografía}

Almeida, M. D. (2009). Rigideces fiscales en Ecuador. Santiago de Chile. 
Asamblea Constituyente. (2008). Ley orgánica para la recuperación del uso de los recursos petroleros del Estado y racionalización administrativa de los procesos de endeudamiento.

Banco Central del Ecador. (2016). Estadísticas Macroeconómicas Presentación Coyuntural.

Buiter, W. H. (1977). "Crowding out" and the effectiveness of fiscal policy. Journal of Public Economics, 7(3), 309-328. http://doi.org/10.1016/0047-2727(77)90052-4

Cetrángolo, O., Pablo, J., \& Compiladores, J. (2009). Documento de proyecto Rigideces y espacios fiscales en América Latina.

Cuello, R. E. (1999). El neoliberalismo, una ideología contraria al equilibrio social. Buenos Aires.

Cuesta Caza, J. A. (2013). La política fiscal implementada en Ecuador y su relación con los modelos alternativos de desarrollo (2007-2012).

De Grauwe, P. (2010). The Return of Keynes. International Finance, 13(1), 157-163. http://doi.org/10.1111/j.1468-2362.2010.01256.x

Galarza, E. (1997). Keynes frente al pensamiento único. Príncipe de Viana. Suplemento de Ciencias Sociales, 16, 21-38.

Giraldo, C. R. (2007). La Eliminación De Los Fondos Petroleros: Explicación Bajo Modelos De Garbage Can Y Policy Window.

Gutiérrez, E. (2008). De las teorías del desarrollo al desarrollo sustentable. Ingenierías, XI(25), 21-35.

Horton, M., \& El-Ganainy, A. (2012). Fiscal Policy: Taking and Giving Away. IMF (Finance and Development), 1-4.

Houben, H. (2011a). A Marxist Critic On The Keynesian Analysis Of The Economic Crisis. Source: World Review of Political Economy, 2(2), 219-234.

Houben, H. (2011b). A Marxist critic on the Keynesian Analysis of the economic crisis. World Review of Political Economy, 2(2), 219-234.

Leijonhufvud, A. (1987). ¿Qué le ha sucedido a la Economía Keynesiana? Estudios Económicos, $2(1(3)), 65-79$.

López Arévalo, J., \& Peláez Herreros, Ó. (2015). El desigual impacto de la crisis económica de 2008-2009 en los mercados de trabajo de las regiones de México: La frontera norte frente a la región sur. Contaduria $Y$ Administracion, 60, 195-218. http://doi.org/10.1016/j.cya.2015.05.004 
Márquez Covarrubias, H. (2016). La gran crisis del capitalismo neoliberal. Andamios, 7(13), 5784.

Montoya, C. A. (2009). Keynes y Neoclásicos: Una propuesta para la salidade la crisis. Revista Ciencias Estratégicas, 17(21), 89-104.

Novelo, F. (2016). La pertinencia actual de la Teoría General de Keynes. Economía UNAM, 13(38), 41-60. http://doi.org/10.1016/j.eunam.2016.05.002

Pérez, E. (2003). Chicago, Keynes and Fiscal Policy. Investigación Económica, 62(246), 15-45.

Secretaría Nacional de Planificación y Desarrollo. (2013). Plan Nacional Buen Vivir 2013-2017.

Streeten, P. P. (2016). Keynes And The Classical Tradition. Revista de Economía Institucional, 5(9), 166-186.

Venegas, C., \& Pilar, S. del. (2010). La crisis económica de 2008. Algunas reflexiones teóricas a partir de J.M. Keynes y H. P. Minsky, 8, 93-114. 